\title{
High resolution multiposition Thomson scattering for the TEXTOR tokamak
}

\author{
C. J. Barth, ${ }^{a}$ H. J. v. d. Meiden, T. Oyevaar, and N. J. Lopes Cardozo \\ FOM-Instituut voor Plasmafysica "Rijnhuizen," Association EURATOM-FOM, Trilateral Euregio Cluster, \\ P.O. Box 1207, 3430 BE Nieuwegein, The Netherlands
}

(Presented on 21 June 2000)

\begin{abstract}
In this article, the recently installed high resolution multiposition Thomson scattering (TS) system of the TEXTOR tokamak is presented. Light from a pulsed ruby laser is scattered by the free electrons of the plasma and transmitted by fiber optics to a polychromator for spectral analysis. The Doppler broadened spectrum of the scattered light is analyzed with a Littrow spectrometer, detected with an image intensifier, and recorded with two intensified charge coupled device cameras. Values of the electron temperature $\left(T_{e}\right)$ in the range of $50 \mathrm{eV}$ up to $4 \mathrm{keV}$ can be measured at 450 spatial elements of $2 \mathrm{~mm}$ along a chord of $900 \mathrm{~mm}$, with a resolution of $8 \mathrm{~mm}$. The observational error on $T_{e}$ was found to be $<3 \%$ at an electron density $\left(n_{e}\right)$ of $3.5 \times 10^{19} \mathrm{~m}^{-3}$ using a laser energy of $\sim 8$ J. These features make - to our knowledge - the multiposition TS system of TEXTOR to be the one with the highest spatial and spectral resolution in the world. In this article the first results of this powerful diagnostic will be presented. (C) 2001 American Institute of Physics. [DOI: $10.1063 / 1.1322602]$
\end{abstract}

\section{INTRODUCTION}

The Torus Experiment for Technology Oriented Research (TEXTOR) is a tokamak with a major plasma radius of $1.75 \mathrm{~m}$ and a minor radius of $0.46 \mathrm{~m}$, with a toroidal magnetic field on axis of $2.6 \mathrm{~T}$ and $I_{p} \leqslant 500 \mathrm{kA}$. The Dutch Thomson Scattering (TS) team of FOM Rijnhuizen has designed and built a multiposition TS system for TEXTOR in cooperation with the IPP staff. This TS system is a further improvement of the TS system used on the former Rijnhuizen Tokamak Project (RTP) ${ }^{1}$ and of the system in operation on the TJ-II stellarator. ${ }^{2}$ The multiposition recording of these so-called TV systems is based on the use of two-dimensional charge coupled device (CCD) cameras, like on the previous Tokamak Fusion Test Reactor ${ }^{3}$ and the present JFT-2M ${ }^{4}$ and Mega-Ampere Spherical Tokamak (MAST) ${ }^{5}$ systems. Contrary to the systems of RTP and TJ-II, fiber optics was applied instead of conventional optics to guide scattered light from the plasma to the polychromator and the detection system outside the tokamak area. This modification was necessary to protect the vulnerable detection system against the high x-ray and neutron levels inside the biological shield.

The TS system can be split up into the laser beam, the detection, and the data acquisition subsystems, which are described in Sections II, III, and VI. The performance of the complete system is presented in Sec. IV, calibration, in Sec. $\mathrm{V}$, and first results in and Sec. VII.

\section{LASER BEAM PATH}

In Fig. 1 the schematic layout the of the complete TS system is shown. Light of a $Q$-switched ruby laser is injected into the plasma along a vertical line. Long entrance and exit tubes containing a series of light baffles are used to suppress

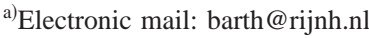

the vessel stray light. Carbon tiles covering the inner liner of the tokamak only serve as a viewing dump for the central part of the laser chord.

The collected scattered light from a laser chord of 900 $\mathrm{mm}$ is transmitted via a fiber array over a distance of $28 \mathrm{~m}$ to a Littrow polychromator for analysis and detection. The ruby laser, a Lumonics PDS2 system, consists of a single-mode oscillator cavity and three amplifier stages, giving an output pulse of $\leqslant 25 \mathrm{~J}$ during $15 \mathrm{~ns}$ full width at half maximum (FWHM) at $694.3 \mathrm{~nm}$, or twice $12.5 \mathrm{~J}$ in double pulse operation. The beam divergence is very low: $90 \%$ of the energy is kept within $0.4 \mathrm{mrad}$. Three motor-controlled $90^{\circ}$ mirrors of $3 \mathrm{in}$. diameter are used to guide the laser beam to the entrance tube along a path of $18 \mathrm{~m}$. The entrance and exit tubes have lengths of 4.0 and $3.7 \mathrm{~m}$, respectively. The beam diameter at both windows is equal to $\sim 32 \mathrm{~mm}$, resulting in an average energy density of $3 \mathrm{~J} / \mathrm{cm}^{2}$ at $25 \mathrm{~J}$ output, which is sufficiently lower than the safety limit of $5 \mathrm{~J} / \mathrm{cm}^{2}$. This additional safety factor was taken into account because of the slightly hollow beam profile in the far field. The laser beam is focused by a telescope $(f=4.3 \mathrm{~m})$ to a spot of $\sim 1.5 \mathrm{~mm}$ at the center of the observational chord. Due to the small divergence of the beam in the vessel $(\sim 8 \mathrm{mrad})$ the laser beam diameter is $\sim 5 \mathrm{~mm}$ at the ends of the observational chord.

Behind the output window the laser beam is dumped by a neutral glass filter (Schott RG 1000) placed under $45^{\circ}$. A beam splitter reflects a small fraction $\left(<10^{-4}\right)$ of the beam into a small integrating sphere, where some light is picked up by a fiber. The exit face of this laser monitor fiber is imaged onto the intensified CCD cameras (Fig. 2, item 12) to make a relative measurement of the laser energy.

A small fraction $(\sim 0.2 \%)$ of the laser oscillator beam is led via a fiber to the spectrometer to trigger the gate pulse of the image intensifier power supply. In this way the intensifier can be gated open $\sim 5-10 \mathrm{~ns}$ before the scattered light arrives at the cathode of the image intensifier, including an 


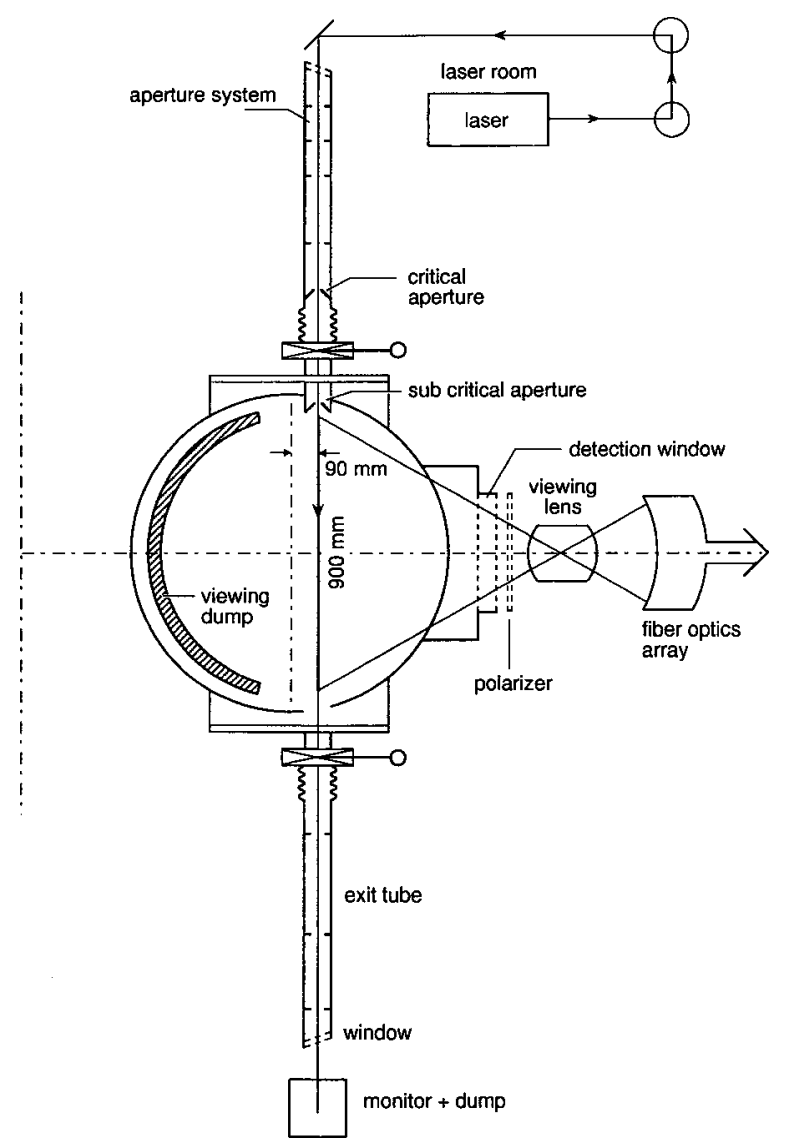

FIG. 1. Layout of the TEXTOR Thomson scattering system.

intrinsic delay of $\sim 15 \mathrm{~ns}$ in the gate pulse unit. As a result of this optical synchronization the gate width could be kept as short as $40 \mathrm{~ns}$, independent of laser energy and jitter.

\section{DETECTION SYSTEM}

In Fig. 2 the basic elements of the detection branch are shown: relay optics, polychromator, and detection system. Scattered light is collected by a six-element viewing lens (Fig. 1) after passing a quartz vacuum window. During wall conditioning this window is protected by a mechanical shutter. A polarizer is used to suppress plasma and vessel stray light by a factor of 2 . The laser beam is imaged by a six element lens system ${ }^{6}$ onto the entrance head of a fiber bundle, ${ }^{7}$ with a magnification of $M_{\text {view }}=0.152$. The fiber array consists of 30 separate bundles with flat entrance and exit

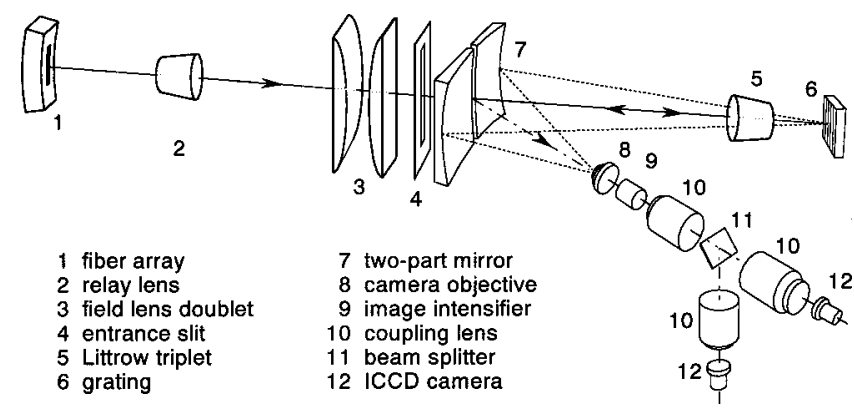

FIG. 2. Layout of the detection branch of the TEXTOR multiposition Thomson scattering system. surfaces. Each bundle consists of $20 \times 3$ fibers $(0.21$ core and $0.23 \mathrm{~mm}$ cladding diameter), sorted in both directions. At the input and output side these bundles are arranged along an arc and such that their principal rays are perpendicular to the curved image $(R=192.8 \mathrm{~mm})$ and object $(140.42 \mathrm{~mm})$ planes, respectively. At the output side the height of the fiber array is reduced and the width is increased by a factor of 2 , thus enlarging the solid angle of the viewing lens by a factor of 4. A seven-element lens system (Fig. 2, item 2) images the fiber output onto the entrance slit of the spectrometer, with a magnification of $M_{\text {relay }}=3.87$. A doublet field lens (item 3) images the exit pupil of the relay lens at the grating surface. Although this field lens has a negative effect on the field curvature the spot size at the entrance slit is still rather small $(<0.05 \mathrm{~mm}$ FWHM along the full slit height).

Spectral analysis is performed by a flat grating positioned in the Littrow setup (item 6). This configuration enables the use of a rather large linear etendue without introducing vignetting losses. In our case a slit height of $260 \mathrm{~mm}$ is combined with a full angle of $85 \mathrm{mrad}$. The entrance slit (item 4) has a width of $4 \mathrm{~mm}$ and is located just behind the two-part spectral mirror (item 7) with a central gap of 11 $\mathrm{mm}$. Collimation of input light and focusing of diffracted light are performed by a Littrow triplet 1:10/948 mm (item $5)$. The grating is oriented such that light at the laser wavelength returns along its original path. In this way vessel stray light is suppressed (see Sec. IV). The spectrum is projected onto a two-part spherical mirror $(R=-780 \mathrm{~mm})$ which serves for pupil imaging. Finally, the spectral image at the mirror of $260 \times 200 \mathrm{~mm}^{2}$ is imaged at the cathode of the detector by a camera objective (Canon 1.0/50 mm) using a magnification of 0.09 . The resulting rectangular image (of $23.4 \times 18 \mathrm{~mm}$ ) is cut off at the four edges since the image intensifier only accepts a diameter of $25 \mathrm{~mm}$. As a result the usable mirror width at the extreme $z$ positions is only 100 $\mathrm{mm}$. This will be no problem since these mirror parts receive scattered light from the plasma edge which normally has low temperatures. The spectral range of the spectrometer runs from 585 to $800 \mathrm{~nm}$.

Detection of the scattered light is performed by a Generation III extended-blue GaAsP image intensifier (item 9) with a diameter of $25 \mathrm{~mm} .{ }^{8}$ The cathode has a quantum efficiency of $\sim 30 \%$ at $700 \mathrm{~nm}$, while the tube efficiency including the microchannel plate noise amounts to $\eta_{\text {intensifier }}$ $\sim 18 \%$. A Rodenstock 1.2/95 mm objective, a 50\% beamsplitter in combination with two $0.95 / 64 \mathrm{~mm}$ objectives (items 10 and 11) are used to image the P20 output screen of the GEN III image intensifier onto the cathodes of two intensified CCD cameras. These two Instaspec V cameras (Andor Technology) combine a $25 \mathrm{~mm}$ Generation II image intensifier (quantum efficiency of $\sim 15 \%$ and a noise factor of $\sim 2$ ) and a 1.5:1 fiberoptic taper with a CCD of $576 \times 385$ pixels each $22 \mu \mathrm{m}$ large $\left(12.67 \times 8.47 \mathrm{~mm}^{2}\right)$. The coupling efficiency of the Rodenstock tandem system and the beamsplitter results in a $5 \%$ yield for each detector branch. The intensifiers of the intensified CCD cameras are gated open during $\sim 1 \mathrm{~ms}$ for single instance observation and during 20 $\mu$ s for double pulse measurements. ${ }^{9}$

The residual longitudinal chromatic aberrations of the 
Canon objective (Fig. 2, item 8) result in a small image tilt of $0.0133 \mathrm{rad}$ along the wavelength direction. This tilt is corrected for by a corresponding rotation of the complete detection system behind the Canon such that the cathode of the GEN III image intensifier coincides with the image plane of the Canon. Light picked up by the laser monitor fiber is imaged via an achromatic doublet (not shown in Fig. 2), two of the Rodenstock lens systems, and the beamsplitter at the cathode of both intensified CCD cameras.

\section{OPTICAL PROPERTIES}

In this section we summarize the most important optical properties of the system: the overall transmission, the resolution, and the stray light ratio of the spectrometer.

The overall transmission of $15 \%$ was estimated from the transmission values of the individual components: quartz window $(92 \%)$; relay system (including viewing lens, fiber, and relay lens: $50 \%$ ), Littrow spectrometer $(50 \%)$ and Canon objective $(65 \%)$. Note, the high transmission of the relay system, which includes 28 antireflection-coated lens surfaces and $28 \mathrm{~m}$ fiber. In- and out-coupling losses of the fiber heads were decreased by the application of coated contact lenses. The contact surface of these lenses has the same curvature as the fiber heads. Optical contact is realized by means of fiber coupling gel. In case of the output side of the fiber array, this contact lens also serves as a field corrector.

The spatial and spectral resolutions of the system have been determined using ray tracing results for the viewing and spectrometer optics together with the measured resolution of the Canon objective. The final spot sizes at the position of the CCD chip for a narrow spectrometer entrance slit of $<0.1 \mathrm{~mm}$ are estimated to be $70 \mu \mathrm{m}$ in the spatial and 60 $\mu \mathrm{m}$ in the wavelength direction along the full image field. Including a CCD resolution of 2 pixels these spots correspond to 3.8 and 3.4 pixels, respectively. The measured spatial resolution was found to be $\sim 4$ pixels, corresponding to 8 $\mathrm{mm}$ in the plasma; while the spectral resolution appeared to be $\sim 3.5$ pixels, corresponding to $2.2 \mathrm{~nm}$. However, the spectral resolution for a slit width of $4 \mathrm{~mm}$ equals 7 pixels, giving a channel width of $4.5 \mathrm{~nm}$.

A laser diode $(670 \mathrm{~nm})$ and a set of diffusers in front of the viewing triplet result in a rather homogeneous illumination of the full slit height. This source was used to measure the stray light ratio of the spectrometer for a grating orientation where the wavelength of the laser diode returns into the slit again. A typical stray light ratio of $\sim 10^{-5}$ adjacent to the central gap, decaying to $\sim 10^{-6}$ at the edge of the spectrum, was found. Therefore, a spectral element close to the gap records $\sim 10^{-5}$ of the total vessel stray light entering the spectrometer. Because of the large vessel stray light level (see Sec. V) a notch filter is placed in front of the camera objective (Fig. 2, item 8) to reach an additional suppression of $10^{-2}-10^{-5}$ depending on the angle of incidence. The transmission outside the notch area of $20 \mathrm{~nm}$ is larger than $85 \%$. In this way the residual stray light level was kept well below the CCD noise level of 2 counts.

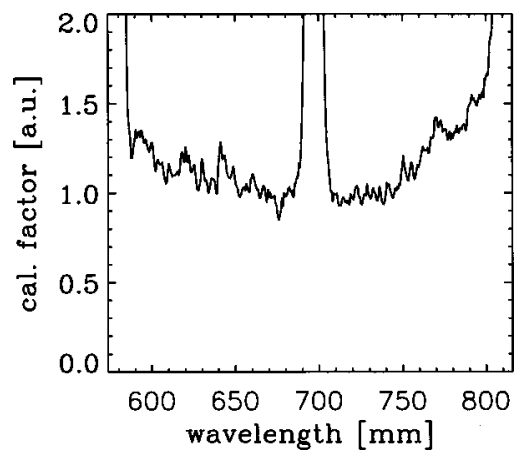

FIG. 3. Relative calibration factors for $z=0 \mathrm{~mm}$.

\section{CALIBRATION}

For the wavelength calibration of the spectrometer He and Ar spectral lamps with suitable lines at 587.56, 667.82, 706.52 , and $764.46 \mathrm{~nm}$ are used. Relative calibration of the system is performed with a tungsten lamp $(T=2685 \mathrm{~K})$ together with an integrating sphere in front of the viewing triplet. The typical calibration curve is rather flat, after normalization to $\sim 1$ at the laser wavelength it increases to 1.5 at the edges of the spectral range (Fig. 3).

The correspondingly decrease in sensitivity of the system at the edge of the spectral range is ascribed to vignetting, the grating efficiency, and the quantum efficiency of the image intensifier.

Rayleigh scattering on nitrogen was used to make an absolute calibration of the system. An attenuator in the laser beam and a rather high filling pressure $(p=16 \mathrm{mbar})$ were used to perform Rayleigh scattering measurements against a rather large vessel stray light level (see Fig. 4).

From these measurements the absolute sensitivity was found to be 10850 at the plasma center, decreasing to $\sim 8000$ counts/pixel J Torr $\mathrm{N}_{2}$ at $z= \pm 450 \mathrm{~mm}$. This decrease in Rayleigh signal shows that the vignetting of the complete system is $\sim 30 \%$, which is partly ascribed to the viewing and relay optics and partly to the Canon objective (15\%).

The number of the detected photoelectrons can be calculated from the scattering formula

$$
N_{\text {pe }}=\frac{E_{\text {laser }}}{h v_{0}} \Delta L \Omega n_{e} \sigma_{T} \tau_{\text {overall }} \eta_{\text {intensifier }} \eta_{\text {slit }},
$$

where the symbols and their values are defined in Table I.

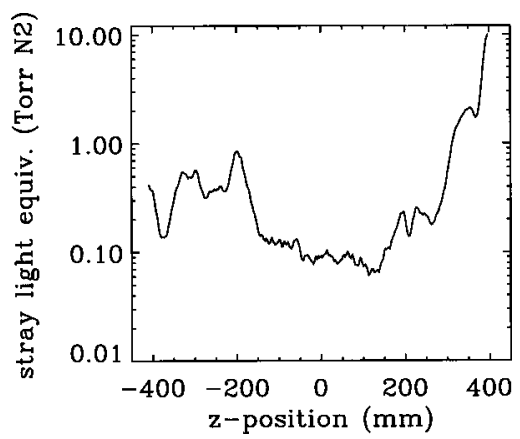

FIG. 4. Vessel stray light equivalent (in Torr $\mathrm{N}_{2}$ ) along the observational chord. 
TABLE I. Summary of the system parameters used to calculate the number of detected photoelectrons for Rayleigh scattering.

\begin{tabular}{lllc}
\hline \hline Symbol & \multicolumn{1}{c}{ Description } & Quantity & Unit \\
\hline$E_{\text {laser }}$ & Laser energy at the scattering volume & 0.88 & $\mathrm{~J}$ \\
$h v$ & Photon energy & $2.86 \times 10^{-19}$ & $\mathrm{~J}$ \\
$\Delta L$ & Length of the sampling volume (4 pixels) & $8.0 \times 10^{-3}$ & $\mathrm{~m}$ \\
$\Omega$ & Solid angle & $1.88 \times 10^{-3}$ & $\mathrm{sr}$ \\
$\tau_{\text {overall }}$ & Overall transmission & 0.15 & \\
$\eta_{\text {intensifier }}$ & Efficiency of the image intensifier & 0.18 & \\
$\sigma_{T}$ & Differential Thomson cross section & $7.94 \times 10^{-30}$ & $\mathrm{~m}^{2} / \mathrm{sr}$ \\
$n_{e}$ & Equivalent electron density for 1 Torr $N_{2}$ & $8.70 \times 10^{19}$ & $\mathrm{~m}^{-3}$ \\
$\eta_{\text {slit }}$ & Throughput of the slit & 0.9 & \\
$N_{\text {pe }}$ & Number of photoelectrons & 781 & \\
\hline \hline
\end{tabular}

The number of photoelectrons is normalized to a laser energy of $1 \mathrm{~J}$ of which about $90 \%$ reaches the scattering volume. The length of the scattering volume was taken equal to the FWHM of the $z$ resolution. From Table I we find $N_{\text {pe }}=781$ photoelectrons/count Torr $\mathrm{N}_{2}$. Together with the Rayleigh signal (summed over 4 pixels) one finds the conversion factor for the complete detector of 68 counts/ photoelectron. For a typical plasma density of 2.5 $\times 10^{19} \mathrm{~m}^{-3}$ and a laser energy of $10 \mathrm{~J}$ we expect $N_{\text {pe }}$ $=2200$ photoelectrons for each spatial element, giving a relative error for the density of $\sim 2 \%$.

\section{DATA HANDLING}

The signals of the intensified CCD cameras are sent to a VME based work station and stored into a data base. Raw signals are corrected for detector offset and optical distortion introduced by the tilt of the two-part mirror. After application of the wavelength and position calibration the signals are multiplied with the relative sensitivity calibration factors. Values of $T_{e}$ and $n_{e}$ are determined by fitting the full relativistic spectral distribution ${ }^{10}$ to the scattering spectra.

\section{FIRST RESULTS}

After five months of installation-spread over $1 \mathrm{yr}$ - the first high resolution TS results on TEXTOR were obtained in March 2000. In Fig. 5 a typical spectrum of one spatial element $(\Delta z=2 \mathrm{~mm})$ is shown. In Fig. $6 T_{e}, n_{e}$, and $p_{e}$ profiles sampled with 445 spatial points are displayed. The relative errors on $n_{e}$ and $T_{e}$ for a $T_{e}$ range of $0.2-1.7 \mathrm{keV}$ are $3 \%$ and $5 \%$, respectively. Using Eq. (1) inserting an $\left\langle n_{e}\right\rangle$ $\sim 3.5 \times 10^{19} \mathrm{~m}^{-3}$, the statistical error on $n_{e}$ is expected to be $\sim 2 \%$, showing that the system performance agrees well with the design values. During the first two months of operation the high resolution TS system, observations of filaments, transport barriers, and magnetic islands were done. Of the latter a nice example is displayed in Fig. 6, even showing the density profile within the magnetic island resolved in 75 spatial points.

\section{DISCUSSION}

After 2 yrs of design, construction, and installation the high resolution multiposition TS system of TEXTOR was successfully brought into operation. The setup of the system is comparable to that of the TJ-II stellarator in operation at

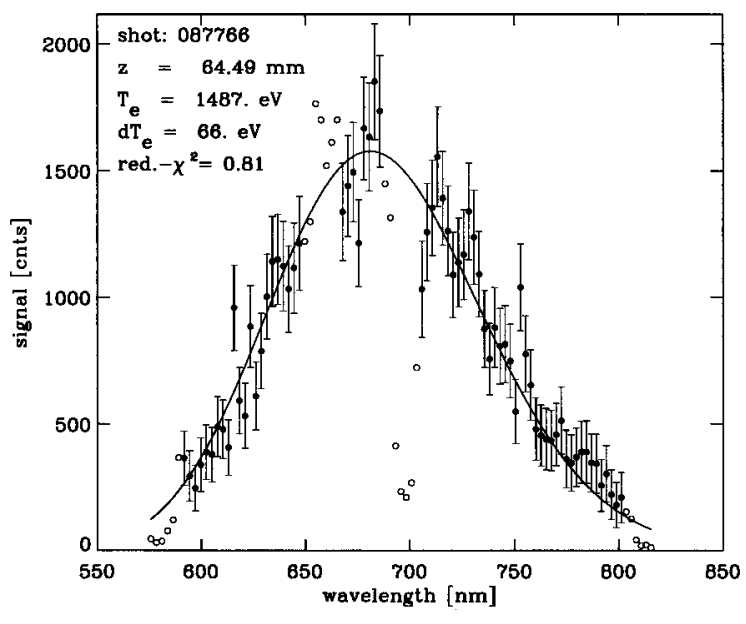

FIG. 5. Thomson scattering spectrum of one spatial element $(\Delta z=2 \mathrm{~mm})$ at $n_{e} \sim 7 \times 10^{19} \mathrm{~m}^{-3}$. Dots refer to spectral points used for the fit, open circles to unused spectral points. Note, the low level of $H_{\alpha}$ light at $653 \mathrm{~nm}$. Laser energy of $8.4 \mathrm{~J}$.

CIEMAT (Madrid) since 1998. The most remarkable difference between the TJ-II and the TEXTOR systems is the application of sorted fibers to relay the scattered light from the plasma device outside the biological shield to the spectrometer. Although the transmission of the TEXTOR system
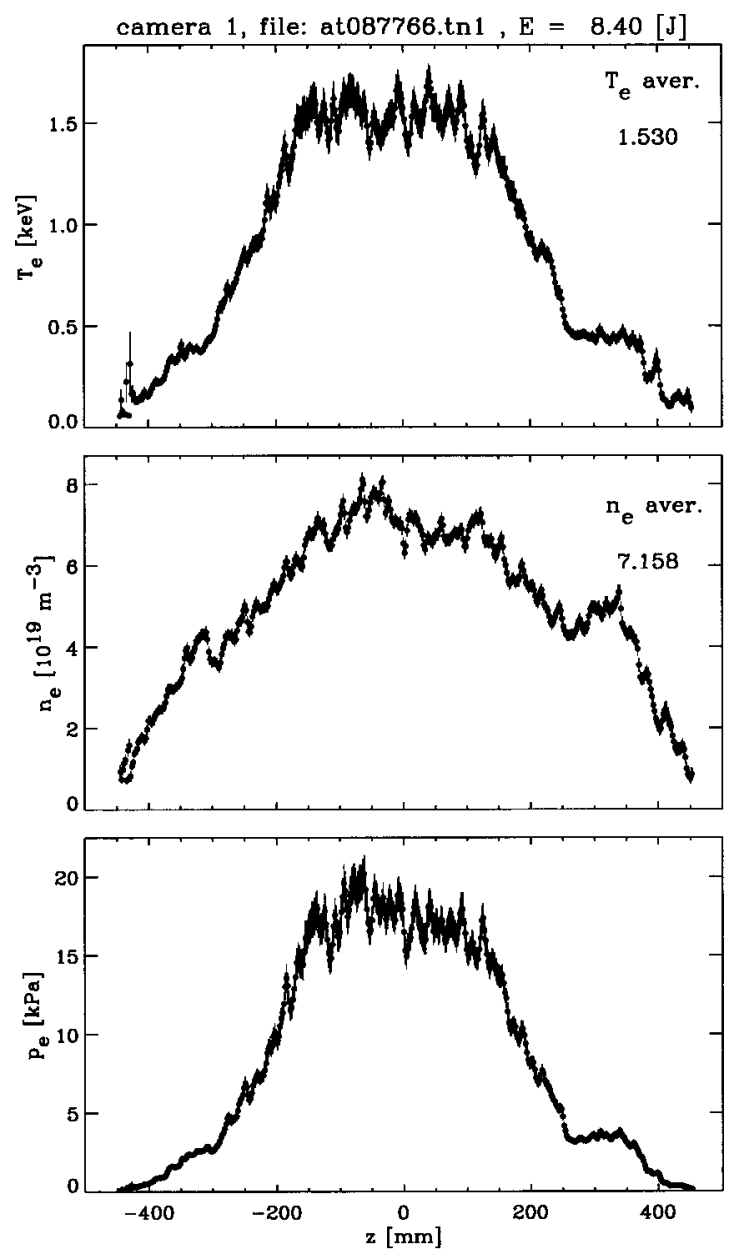

FIG. 6. $T_{e}, n_{e}$ and $p_{e}$ profiles sampled with 445 spatial points, showing distinct $m / n=2 / 1$ magnetic islands at $z \sim \pm 350 \mathrm{~mm}$. Clearly, the density profiles within the magnetic island can be seen. Laser energy of $8.4 \mathrm{~J}$. 
$(15 \%)$ is twice as low as that of the TJ-II system (33\%), the net signal yield is only $15 \%$ less. This can be understood from the larger scattering volume $(2.5 \times)$ and the smaller solid angle $(0.72 \times)$. The first results stress that high resolution TS is an indispensable tool for the research of thermonuclear plasmas.

\section{ACKNOWLEDGMENTS}

The authors are much indebted to their colleagues of the drawing office, especially Koos van Leusden, and of the mechanical divisions at the Forschungs Zentrum Jülich and at FOM Rijnhuizen. For the construction of the fiber array we could rely on the experience of Wim Mastop. During the installation lots of thankful support was given by other members of the TEXTOR team. Data acquisition would have been impossible without the help of Peter Busch and other members of the Software Engineering Group. This work was performed as part of the research program of the association agreement of Euratom and the "Stichting voor Fundamen- teel Onderzoek der Materie"' (FOM) with financial support from the "Nederlandse Organisatie voor Wetenschappelijk Onderzoek', (NWO) and Euratom.

${ }^{1}$ C. J. Barth, M. N. A. Beurskens, C. C. Chu, A. J. H. Donné, N. J. Lopes Cardozo, J. Herranz, H. J. v. d. Meiden, and F. J. Pijper, Rev. Sci. Instrum. 68, 3380 (1997).

${ }^{2}$ C. J. Barth, F. J. Pijper, H. J. v. d. Meiden, J. Herranz, and I. Pastor, Rev. Sci. Instrum. 70, 763 (1999).

${ }^{3}$ D. Johnson, N. Bretz, D. Dimock, B. Grek, D. Long, R. Palladino, and E. Tolnas, Rev. Sci. Instrum. 57, 1856 (1986).

${ }^{4} \mathrm{~T}$. Yamauchi and TVTS team, in 20th EPS Conference on Controlled Fusion and Plasma Physics, Lisboa 1993, Vol. III, p. 1195.

${ }^{5}$ M. J. Walsh, N. J. Conway, M. Dunstan, M. J. Forrest, and R. B. Huxford, Rev. Sci. Instrum. 70, 742 (1999), describing the previous START system, which has recently been installed on MAST.

${ }^{6}$ Designed by RBH-optics.

${ }^{7}$ Manufactured by Fiberguide Industries.

${ }^{8}$ Manufactured by the former Intevac, the present Litton.

${ }^{9}$ M. N. A. Beurskens, C. J. Barth, N. J. Lopes Cardozo, and H. J. van der Meiden, Plasma Phys. Controlled Fusion 41, 1321 (1999).

${ }^{10} \mathrm{M}$. Mattioli, Incoherent light scattering from high temperature plasmas (EUR-CEA-FC-752, 1974). 